\title{
Constellation Design in Four Dimensions under Average Power Constraint
}

\author{
Farbod Kayhan, Nicolò Mazzali and Bhavani Shankar M.R. \\ Interdisciplinary Centre for Security, Reliability and Trust (SnT), \\ Weicker Building, University of Luxembourg \\ 4, rue Alphonse Weicker, L-2721 Luxembourg \\ (e-mail: farbod.kayhan, nicolo.mazzali, bhavani.shankar@uni.lu)
}

\begin{abstract}
In this paper we address the problem of constellation design in four dimensional space (4D) under average power constraint. We compare the performance of best lattice constellations with those optimized without any constraint on their structure. Even though the lattice based constellations provide the best minimum distance, usually they do not allow for a good binary labelling. Therefore, in more realistic system scenarios, up to a $2 \mathrm{~dB}$ gain can be obtained by properly optimizing the constellation and the corresponding binary labelling. We also investigate the performance of $4 \mathrm{D}$ constellations obtained by the Cartesian product of two 2D constellations.

Index Terms-Multidimensional Constellations, Lattice Based Constellations, Shaping Gain, Polypolarization Multiplexing
\end{abstract}

\section{INTRODUCTION}

Multidimensional constellations have been proposed to enhance spectral efficiency in many systems. For example, systems with more than one polarization, multidimensional coded modulation systems and systems where only a fraction of bits is needed in two dimensions. It is well known that constellation enlargement can allow a denser packing of the constellation points for a given minimum Euclidean distance and average power [1]. This would, in turn, enhance the spectral efficiency of the system.

In general, the multidimensional constellations have better figures of merit than 2D constellations. As a case in point, the QAM constellations suffer a loss from the promised Gaussian capacity of $1.53 \mathrm{~dB}$. This so called shaping loss is mainly due to the finite size and the shape of the QAM constellations. However, such a loss can be partially compensated by using multidimensional constellations based on lattices. Indeed, in four dimensional space (4D), the asymptotic shaping gain provided by a lattice-based design is only $0.46 \mathrm{~dB}$ [2]. The densest lattice in 4D is known, and its characteristics have been thoroughly analyzed in [3]. Nevertheless, optimal latticebased constellations may perform poorly in practical systems for several reasons. First of all, these constellations optimize only the minimum Euclidean distance of the constellation which is a good design criterion only if the signal-to-noise ratio (SNR) is very large. The second problem is cutting out a finite and bounded subset of the lattice. In particular, in binary digital systems where it is desired to associate $m$ bits to each symbol, we need to choose $2^{m}$ vertices of the lattice. This would imply some loss in the performance over non periodic channels. In such systems, also the labelling becomes an issue, as it is not possible to find a Gray labelling for constellations with size $M$ where at least one signal has more than $\log _{2} M$ neighbours. Some labelling procedures have been proposed in [4] and references therein, but no performance assessment in a realistic scenario was shown. As we will see in this paper, in BICM systems, this may cause a performance degradation in excess of $2 \mathrm{~dB}$.

Despite the problems mentioned above, the multidimensional constellation design has been subject of many investigations in the last few years and several techniques exploiting more than one polarization have been proposed. For example, the authors in [5] introduce the poly-polarization multiplexing (PPM) technique. In the same paper, also the constellation design for PPM has been discussed.

Moreover, several schemes exploiting more than one polarization have been proposed for optical communications. See for example [6] and some references within. However, in all of them only small size QAM or PSK constellations have been considered, and no particular further design has been proposed.

Our goal in this paper is twofold. First, we optimize the 4D constellations and the corresponding labelling by maximizing the pragmatic achievable mutual information and compare their performance with the best lattice based constellations. Second, we optimize the 2D constellations and compare their performance with those optimized in 4D. To have a fair comparison, we generate a 4D constellation from the optimized 2D points as a Cartesian product. The average power of all constellations are normalized to unity in 4D. As we will see, the proposed optimized constellations perform better than the known constellations in the literature. We are mainly interested in 64-ary constellations in this paper. We have also optimized the 16-ary constellations, but the gain that can be obtained with respect to the Cartesian product of two 4-QAM is negligible. Notice that there are exactly sixteen quadrants in the 4D space and therefore $4 \times 4$-QAM has exactly one point in each quadrant. In other words, it can be seen as a BPSK constellation in each dimension, which is known to be optimal at very low SNRs.

The rest of this paper is organized as follows. In Section II, we describe some basic concepts and define the objective function to be optimized. In Section III, we briefly review the 
algorithms used to optimize constellations and the labelling. Various constructions for 64-ary constellations are presented in Section IV. The performance comparison and the simulation results for proposed constellations are presented in Section V. Finally, we conclude the paper in Section VI and suggest some possible future improvements.

\section{Basic Notations and Problem Statement}

A constellation $\chi$ is simply a finite subset of the $k$ dimensional Euclidean space, i.e., $\chi \subset \mathbb{R}^{k}$. In this paper, we are mainly interested in constellation with $M=2^{m}$ elements and $k=4$. The elements of $\chi$ are referred to as constellation points, transmitted symbols or simply signals. The symbols are associated to the bits at the input of the modulator through the one-to-one labelling $\mu: \chi \rightarrow\{0,1\}^{m}$. For any given symbol $x$, we denote by $\mu^{i}(x)$ the value of the $i^{t h}$ bit of the label associated to it.

A labelling for $\chi$ is called a Gray labelling if for any two symbols $x_{i}, x_{j} \in \chi$, we have $d_{H}\left(\mu\left(x_{i}\right), \mu\left(x_{j}\right)\right)=1$ if $d\left(x_{i}, x_{j}\right) \leq d\left(x_{i}, x_{k}\right)$, for all $x_{k} \in \chi$, where $d_{H}(.,$. and $d(.,$.$) are the Hamming and Euclidean distance functions$ respectively.

We consider a 4D AWGN channel where the received signal can be written as $y=x+n$, where the AWGN noise $n$, transmitted symbol $x$ and received signal $y$ are all in $\mathbb{R}^{4}$. In practice, usually the 4D constellation is projected onto two 2D planes called polarization. Signals in each polarization are then complex numbers.

\section{A. Objective Function}

Bit interleaved coded modulation (BICM) has been accepted in many standards in recent years [7]. The main idea is to separate the detector from the decoder at the receiver. This allows to substantially reduce the complexity of the receiver. However, such systems suffer a large performance degradation if a good binary labelling $\mu$ is not chosen. In such systems, the so called pragmatic achievable mutual information (PAMI) defined in [7] is usually a good performance measure for constellation design:

$$
I_{p}(\chi, \mu)=\sum_{i=1}^{m} I\left(\mu^{i}(x) ; y\right),
$$

where $I(.,$.$) is the mutual information function and \mu^{i}(x)$ is the random variable indicating the $i^{\text {th }}$ bit associated to the transmitted signal.

On the other hand, when iterations between decoder and detector are allowed, the binary labelling becomes less important and the achievable mutual information (AMI), $I(\chi)=I(x ; y)$ can be used as the quality measure.

\section{B. Problem Statement}

Our problem is to find the constellations $\chi$ and the corresponding labelling $\mu$ that maximize the PAMI, $I_{p}$, under the average power constraint. Therefore, the optimization problem can be formulated as:

$$
\begin{array}{rc}
\arg \max _{(\chi, \mu)} & I_{p}(\chi, \mu), \\
\text { subject to: } & \frac{1}{M} \sum_{u \in \chi}|u|^{2} \leq 1
\end{array}
$$

This optimization problem is known to be hard because the $I_{p}$ function usually allows for many local maxima. Notice that the average power constraint is over the $4 \mathrm{D}$ space and not per polarization.

If the shape of the constellation is fixed a priori, one still needs to find the best possible labelling that maximizes the PAMI. In such cases, the optimization problem is slightly different and same optimization algorithm may not be suitable for both problems.

\section{Constellation Design}

Constellation design problem has been an active field of research since the advent of information theory. The optimal solutions for the problem defined in (1) are not known even for small constellation sets. Usually, the optimization algorithms are trapped in a local maximum of the problem, specially when the constellation set has more than 16 points. Further, the shape of the optimized constellation for a given communication system depends on several factors. The most important parameters affecting the shape are the channel model, the power constraint, the chosen objective function to be optimized, and the optimization algorithm per-se. Several algorithms have been proposed in the literature for this optimization problem. In the following we briefly review the algorithms we use for optimization in this paper.

\section{A. Genetic Algorithm for Constellation Cut and labelling Optimization}

The genetic algorithm (GA) has been already used in the literature for constellation design (see for example [8]). In the following, we specialize it to two distinct optimization problems for 4D lattice-based constellations. The first problem is the selection of a finite number of points from a given (infinite) lattice. Indeed, the average power constraint usually selects a number of points higher than the desired cardinality. Therefore, only a subset of the points satisfying the average power constraint has to be selected. This selection is done by implementing a GA which maximizes the AMI of the selected constellation.

The second problem is to find a good labelling for a given constellation. A genetic algorithm has been implemented for finding the labelling which maximizes the PAMI of the given constellation.

\section{B. Simulated Annealing for Joint Constellation and labelling Optimization}

A simplified simulated annealing (SA) has been introduced for constellation design problem over AWGN in [9] for optimizing small size constellations. This algorithm has been extended for optimizing the constellations with up to 256 


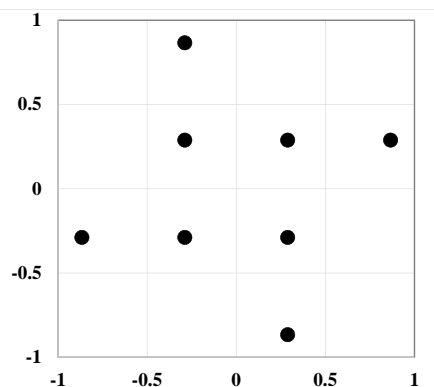

Fig. 1. The 8-QAM constellation used in this paper as the benchmark.

signals under more general channel models in [10]. The main advantage of the SA algorithm with respect to other algorithms in the literature is that it allows to jointly optimize the constellation and the corresponding labelling. This is of great importance because a constellation optimized without considering the labelling may not allow for a Gray mapping, and hence will show a large performance degradation in practical systems. For details on the SA algorithm for constellation design we kindly refer the readers to [10] and the references therein. A straightforward extension of the SA algorithm for the optimization of 4D constellations can be found in [11], [12].

\section{64-ARY CONSTELLATION DESIGN IN 4D}

In this section, we present the 64-ary constellations which are considered in this paper. As mentioned before, the shape of the optimized constellation depends also on the selected SNR. Our target SNR for optimization is $10 \mathrm{~dB}$ which approximately corresponds to an AMI of 5 bits per transmitted symbol. The simplest constellation can be constructed by taking the Cartesian product of two 8-QAM constellations shown in Fig. 1. We denote the resulting constellation as $8 \times 8$-QAM and use it as a benchmark for performance evaluation.

\section{A. Lattice Amplitude Modulation}

Following [13], we refer to the multidimensional latticebased constellations by Lattice Amplitude Modulations (LAMs), where the basic construction procedure involves taking symbols from a suitable 4D lattice. According to the lattice chosen, different types of constellations, with varied properties, can be derived.

The densest packing in $4 \mathrm{D}$ is given by the $D_{4}$ lattice (the so-called checkerboard), which is a cubic lattice centered at the origin. It is obtained by partitioning the quadratic lattice $Z_{4}$ and choosing only the points whose (integer) coordinates sum to 0 (modulo 2). Being the densest lattice in $4 \mathrm{D}$, the $D_{4}$ provides the best coding gain by maximizing the minimum Euclidean distance among the constellation points [3].

In practical systems, the bits at the output of the encoder select the points in the $4 \mathrm{D}$ constellation to be transmitted. However, the pulse shaping and the transmission over the

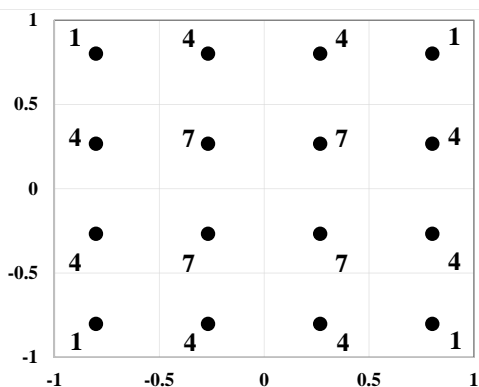

Fig. 2. The 2D projection of the 64-LAM constellation. The number of occurrences for each point in $4 \mathrm{D}$ is reported for each transmitted symbol.

channel normally occur separately per polarization. This implies that each selected $4 \mathrm{D}$ symbol has to be projected on the two 2D planes, one per polarization, before being linearly modulated by means of pulse shaping, and finally transmitted over the channel. Since at a given time instant the projected 2D symbols on each polarization are correlated (because of the shaping introduced by the 4D design [14]), the optimal detector has to operate jointly on both the polarizations.

1) 64-LAM: To obtain the 64-LAM constellation, we first select the smallest sphere around the origin which contains at least 64 vertices of the $D_{4}$ lattice. In this case, the spherical cut selects 88 points of which 40 points have lower amplitudes and the remaining 48 points have the highest amplitude. The final constellation is obtained by selecting all the vertices with lower amplitudes and only 24 points with highest. In order to choose this 24 points, a GA is implemented to maximize the AMI of the constellation. We show the $2 \mathrm{D}$ projection of the resulting constellation in Fig. 2. Also, the number of occurrences for each point is reported in this figure.

2) labelling for 64-LAM Constellation: In the $D_{4}$ lattice, each point has up to 24 neighbours (the number of neighbours is called the kissing number of the lattice). This indicates that having a Gray mapping which allows for 1 bit flip for all the couples of neighbouring points is not possible. Actually, by some combinatorial analysis, it can be shown that even a mapping allowing a difference of 2 bits between neighbouring symbols is still not possible for 64-LAM constellation. Therefore, we have used a GA for finding a labelling which optimizes the PAMI of the constellation.

\section{B. Enhanced Poly-polarization Multiplexing}

The 4D constellation design for enhanced poly-polarization multiplexing (EPPM) has been studied by Yofune et al. in a series of papers (see for example [5] and [15]). For the sake of completeness, we also compare our proposed constellation with those reported in these papers. For more details on these constellations we kindly refer the readers to [5] and the references within. We show the 2D projection of this constellation in Fig. 3. Also, the number of occurrences for each point is reported in this figure. 


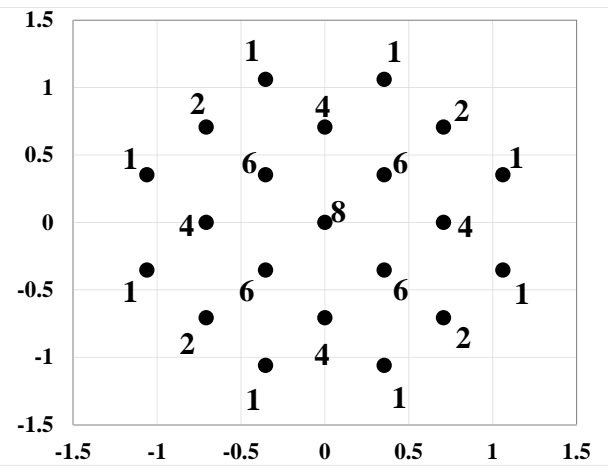

Fig. 3. The 2D projection of the 64-EPPM constellation. The number of occurrences for each point in 4D is reported for each transmitted symbol.

\section{Cartesian Product of Optimized 2D Constellations}

As shown in [14], the 4D design induces a correlation among the 2D symbols obtained by projecting the $4 \mathrm{D}$ constellation onto the two 2D polarization planes. As stated before, the optimal detector is joint over the two polarizations, i.e., it takes into account the correlation between the received samples on both the polarizations when computing the log likelihood ratios. Of course, this increases the overall complexity of the detection procedure. A simpler detector operating separately on the two polarization (i.e., treating the received samples from different polarizations as independent) would have a poorer performance than the optimal detector. However, such a loss can be mitigated by an accurate design of the 2D projected constellation. Indeed, 4D constellations that can be decomposed as a Cartesian product of two 2D constellations have independent projected constellations (which are coincident with the constituting 2D constellations). Of course, designing a 4D constellation as a Cartesian product of two 2D constellations results in a shaping loss.

The main question is how big is the shaping loss if the constellations in 2D are properly optimized. A key objective of this evaluation is to trade-off the shaping loss vis-a-vis decoding complexity when 2D constellations are optimized. We have optimized an 8 point constellation in 2D using the SA algorithm. This constellation is shown in Fig. 4. We denote by $8 \times 8$-OPT the constellation in $4 \mathrm{D}$ obtained by the Cartesian product of this constellation with itself.

\section{Optimized 4D Constellations}

As we have mentioned, the SA algorithm can be easily modified for optimizing the 4D constellations under the average power constraint by maximizing the AMI or PAMI. We have initialized the SA algorithm with the $8 \times 8$-OPT in order to speed up the algorithm. The optimized constellation is shown in Fig. 5. Notice that we show both polarization in a single figure. We refer to this constellation as the 64-OPT in what follows. The target SNR for the optimization is chosen to be 10 $\mathrm{dB}$. The coordinates for the points of this constellation together

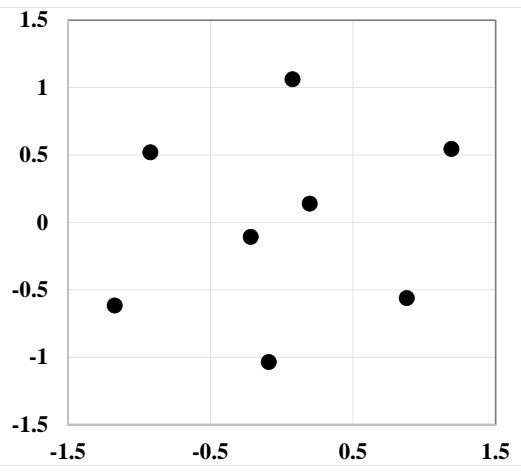

Fig. 4. The optimized 8 points constellation in 2 dimension for $S N R=10$.

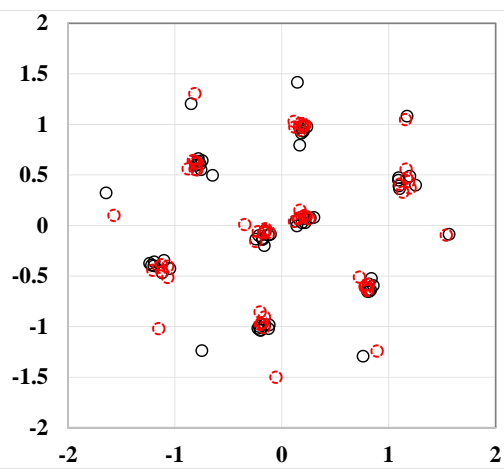

Fig. 5. The optimized 64 points constellation in 4D. The black circles are the coordinates of the first polarization and the red dashed circles are coordinated of the second polarization.

with the corresponding labelling can be found in Appendix A.

\section{Performance Comparison and Simulation RESULTS}

In this section we first plot the AMI and PAMI curves, and then present the BER simulation results for the constellations we have introduced in Section III.

\section{A. AMI and PAMI Comparison for 64-ary Constellations}

In Fig. 6 we show the AMI for 64-ary constellations. For the SNR values in the interval $[6,12]$, the $64-\mathrm{OPT}$ is better than 64-LAM, even though this constellation is designed to optimize the PAMI. For larger SNRs, 64-LAM has a better AMI. This is not surprising, because 64-LAM has a better minimum Euclidean distance, which indeed becomes the dominant factor in AMI computation at high SNRs. However, the interested SNR values are those providing spectral efficiencies between $0.5 \mathrm{~dB}$ to $1.5 \mathrm{~dB}$ below the saturation point. In Fig. 6 , we can also notice that $8 \times 8$-OPT constellation shows a large degradation for SNR values larger than $10 \mathrm{~dB}$. EPPM 


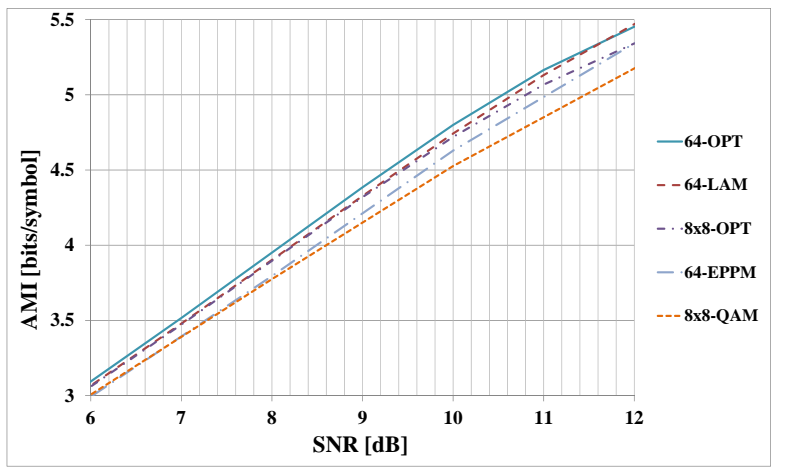

Fig. 6. AMI comparison for 64-ary constellations.

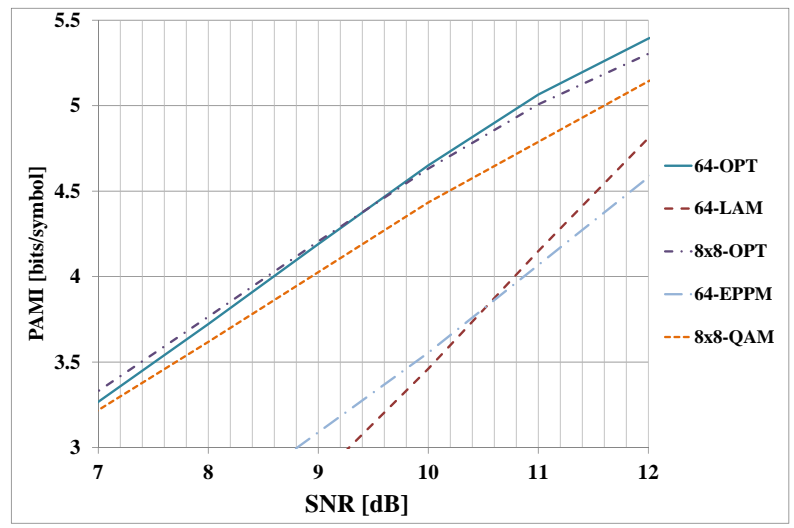

Fig. 7. PAMI comparison for 64-ary constellations.

constellation and $8 \times 8$-QAM show a loss larger than $0.5 \mathrm{~dB}$ with respect to the 64-OPT.

In Fig. 7, we show the PAMI for constellations with 64 signals. Notice that 64-LAM and 64-EPPM constellations suffer a significant loss with respect to their AMI values in the previous figure. This is mainly because a good labelling does not exit for these constellations. The 64-OPT and $8 \times 8$ OPT have almost the same PAMI values at $\mathrm{SNR}=10 \mathrm{~dB}$.

\section{B. Simulation Results}

In this section we provide the bit error rate (BER) simulations for all the 64-ary constellations introduced in the previous section. A GEO satellite broadband system employing both the polarizations for transmission on the user link is considered. Further, a DVB-S2 air interface [16] is assumed and we use the LDPC code of the DVB-S2 standard with rate $R=5 / 6$ and the code length $n=64800$ bits. The channel is AWGN and no cross polarization has been considered. As we can see in Fig. 8, the BER curves confirm closely the PAMI results which were presented in Fig. 7.

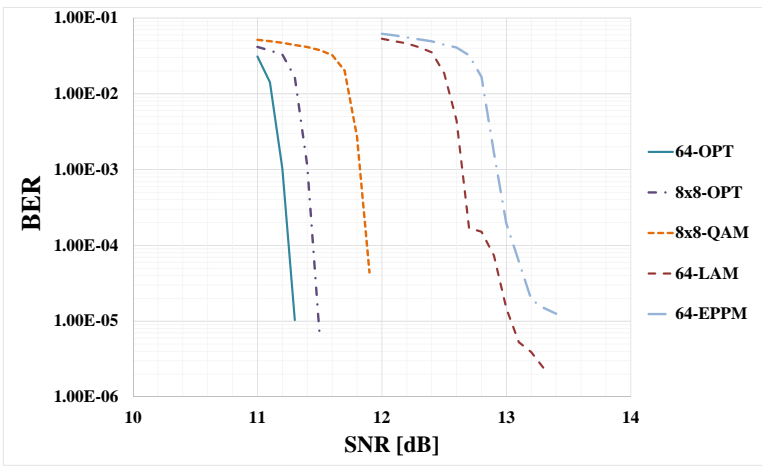

Fig. 8. BER comparison for 64-ary constellations over the AWGN channel.

\section{CONCLUSIONS AND FURTHER STUDIES}

In this paper, we have optimized 64-ary 4D constellations by maximizing the pragmatic achievable mutual information (PAMI). A simulated annealing algorithm has been used to jointly optimize the shape and labelling of the constellation. We have compared the PAMI of optimized constellation with LAM, EPPM, and QAM constellations, showing a gain of at least $0.5 \mathrm{~dB}$ over the AWGN channel. On the other hand, the 4D constellation designed by the Cartesian product of two 2D optimized constellation shows a degradation of only $0.2 \mathrm{~dB}$ with respect to the $4 \mathrm{D}$ optimized constellation, resulting more appealing in systems where the receiver complexity is an issue. In this paper we have limited ourselves to the AWGN channel. The performance assessment of 4D constellations over other channel models where the correlation between the two polarizations can be exploited by a joint detector to improve the performance (e.g., erasure channel, deep fading channel) will be object of future investigations. Another interesting direction for further research is to take into account the cross-polar interference at the constellation design level.

\section{ACKNOWLEDGMENT}

This work was partly funded by the European Space Agency's Satellite Network of Experts (SATNEX IV) initiative, Call-of-Order 1 under the contract 4000113177/15/NL/CLP.

The simulated annealing algorithm used for 4D constellation design in this paper has been implemented by Tianyi Liu and Qizhen Lu.

\section{APPENDIX A}

In Table I we provide the coordinates of the 64-OPT constellation. Notice that the binary labelling for each point is reported in decimal at the beginning of each row. For example, the binary labelling corresponding to the point in row 11 is '001011'. 


\section{REFERENCES}

TABLE I

THE COORDINATE OF THE 64-OPT CONSTELLATION IN 4D.

\begin{tabular}{|c|c|c|c|c|}
\hline 0 & 0.819531 & -0.650599 & 0.783234 & -0.612464 \\
\hline 1 & -0.203173 & -1.032884 & 0.788877 & -0.589036 \\
\hline 2 & 0.141438 & -0.004051 & 0.891761 & -1.24174 \\
\hline 3 & -0.177842 & -0.13861 & 0.725939 & -0.510649 \\
\hline 4 & 1.100741 & 0.364006 & 0.779372 & -0.60412 \\
\hline 5 & -1.195694 & -0.39896 & 0.810181 & -0.583647 \\
\hline 6 & 0.201804 & 0.931051 & 0.802697 & -0.642245 \\
\hline 7 & -0.762647 & 0.607541 & 0.788062 & -0.622796 \\
\hline 8 & 0.831583 & -0.639384 & -0.203313 & -0.97634 \\
\hline 9 & -0.198017 & -1.037786 & -0.189953 & -0.976069 \\
\hline 10 & 0.189457 & 0.027512 & -0.053883 & -1.500724 \\
\hline 11 & -0.181727 & -0.128828 & -0.202543 & -0.855103 \\
\hline 12 & 1.091693 & 0.448302 & -0.162538 & -0.905415 \\
\hline 13 & -1.19357 & -0.359385 & -0.166254 & -1.010295 \\
\hline 14 & 0.193993 & 0.93641 & -0.192896 & -0.967122 \\
\hline 15 & -0.743537 & 0.641533 & -0.181698 & -0.96092 \\
\hline 16 & 0.838133 & -0.523245 & 0.188007 & 0.082647 \\
\hline 17 & -0.747265 & -1.237626 & 0.150552 & 0.067818 \\
\hline 18 & 0.300484 & 0.079948 & 0.170029 & 0.151495 \\
\hline 19 & -0.214198 & -0.09935 & 0.25579 & 0.066814 \\
\hline 20 & 1.564649 & -0.086355 & 0.119476 & 0.040546 \\
\hline 21 & -1.217543 & -0.392582 & 0.16196 & 0.073242 \\
\hline 22 & 0.167983 & 0.794089 & 0.228777 & 0.085232 \\
\hline 23 & -0.647662 & 0.496357 & 0.215085 & 0.099091 \\
\hline 24 & 0.760562 & -1.292667 & -0.15185 & -0.032272 \\
\hline 25 & -0.123657 & -1.020183 & -0.219344 & -0.060701 \\
\hline 26 & 0.218734 & 0.027632 & -0.345939 & 0.009512 \\
\hline 27 & -0.163616 & -0.198903 & -0.243161 & -0.157441 \\
\hline 28 & 1.171474 & 1.0817 & -0.117722 & -0.066364 \\
\hline 29 & -1.6 & 0.322923 & -0.168589 & -0.07312 \\
\hline 30 & 0.146498 & 1.415942 & -0.157298 & -0.063966 \\
\hline 31 & -0.847559 & 1.203975 & -0.154092 & -0.049581 \\
\hline 32 & 0.815139 & -0.577882 & 1.169432 & 0.468911 \\
\hline 33 & -0.11 & -0.9 & 1.105125 & 0.408389 \\
\hline 34 & 0.161394 & 0.060585 & 1.157558 & 1.047462 \\
\hline 35 & -0.106251 & -0.087128 & 1.538374 & -0.094519 \\
\hline 36 & 1.092972 & 0.47274 & 1.132569 & 0.3296 \\
\hline 37 & -1.046511 & -0.425708 & 1.162975 & 557353 \\
\hline 38 & 0. & 68 & 93 & 978 \\
\hline 39 & -0.803395 & 0.594136 & 1.200036 & 0.371563 \\
\hline 40 & 0.855747 & -0.593635 & -1.123004 & -0.38806 \\
\hline 41 & -0.157837 & -0.978909 & -1.205712 & -0.44274 \\
\hline 42 & 0.1 & 0.0 & 174 & 0.100403 \\
\hline 43 & -0.125721 & -0.094366 & -1.150115 & -1.01997 \\
\hline 44 & 1.095818 & 0.391156 & -1.066089 & -0.513468 \\
\hline 45 & -1.117878 & -0.467959 & -1.067192 & -0.403078 \\
\hline 46 & 0.184374 & 0.912687 & -1.149613 & -0.412028 \\
\hline 47 & -0.7 & 34 & -1.1 & -0.458795 \\
\hline 48 & 0.803038 & -0.653412 & 0.169144 & 1.007653 \\
\hline 49 & -0.223387 & -1.016917 & 0.192578 & 0.98524 \\
\hline 50 & 0.266615 & 0.074235 & 0.197939 & 969013 \\
\hline 51 & -0.241956 & -0.134771 & 0.115858 & 1.030041 \\
\hline 52 & 1.251754 & 0.399053 & 0.174276 & 0.943906 \\
\hline 53 & -1.235271 & -0.374179 & 0.122326 & 0.973454 \\
\hline 54 & 0.235401 & 0.976446 & 0.194867 & 1.008063 \\
\hline 55 & -0.778252 & 0.634297 & 0.216943 & 0.994425 \\
\hline 56 & 0.808997 & -0.634665 & -0.827652 & 0.642615 \\
\hline 57 & -0.210636 & -0.999016 & -0.812733 & 0.638088 \\
\hline 58 & 0.212436 & 0.069478 & -0.79449 & 0.550199 \\
\hline 59 & -0.158311 & -0.053973 & -0.812503 & 1.303958 \\
\hline 60 & 1.197888 & 0.487771 & -0.810947 & 0.55627 \\
\hline 61 & -1.101382 & -0.344639 & -0.87537 & 0.560765 \\
\hline 62 & 0.199749 & 0.98353 & -0.794465 & 0.621176 \\
\hline 63 & -0.780847 & 0.661811 & -0.804369 & 0.614651 \\
\hline
\end{tabular}

[1] G.D. Forney and Lee-Fang Wei, "Multidimensional constellations. I. Introduction, figures of merit, and generalized cross constellations",IEEE Journal on Selected Areas in Communications, vol.7, no.6, pp.877-892, Aug 1989. doi: 10.1109/49.29611

[2] R. Laroia, N. Farvardin and S. A. Tretter, "On Optimal Shaping of Multidimensional Constellations", IEEE Trans. Info. Theory, Vol. 40, No. 4, July 1994.

[3] J.H. Conway and N.J.A. Sloane, Sphere Packings, Lattices, and Groups, Springer, New York, ed. 3, 1999.

[4] A. K. Khandani and P. Kabal, "Shaping Multidimensional Signal Spaces-Part II: Shell-Addressed Constellations", IEEE Trans. Inf. Theory, vol. 39, n.6, pag. 1809-1819, Nov. 1993

[5] M .Yofune, J. Webber, K. Yano, H. Ban, and K. Kobayashi, Optimization of Signal Design for Poly-Polarization Multiplexing in Satellite Communications, IEEE Communications Letters, 17.11: (pp. 20172020). 2013.

[6] M. Karlsson, and E. Agrell, "Which is the most power-efficient modulation format in optical links?", Optics express, 17.13 (2009): 10814-10819.

[7] G. Caire, G. Taricco, and E. Biglieri, "Bit-interleaved coded modulation,"IEEE Trans. on Inf. Theory, vol. 44, pp. 927-946, May 1998.

[8] Keqian Yan, Fang Yang, Changyong Pan, Jian Song, Fei Ren and Jia $\mathrm{Li}$, "Genetic algorithm aided gray-APSK constellation optimization," in 9th International Wireless Communications and Mobile Computing Conference (IWCMC), pp.1705-1709, 1-5 July 2013.

[9] F. Kayhan and G. Montorsi, "Joint Signal-labeling Optimization for Pragmatic Capacity under Peak-Power Constraint", IEEE Globecom 2010, Miami, 6-10 Dec. 2010.

[10] F. Kayhan and G. Montorsi, "Constellation design for memoryless phase noise channels", IEEE Transactions on Wireless Communications, vol.13, no.5, pp.2874,2883, May 2014.

[11] T. Liu, "Software Development for Constellation Optimization: Optimization and analysis under average power constraint", Student Project Report, politecnico di Torino, 2015.

[12] Q. Lu, "Software Development for Constellation Optimization: The optimization and analysis under peak power constraint", Student Project Report, politecnico di Torino, 2015.

[13] L. J. Arend, "On dual-polarization signalling techniques in satellite communications", Ph.D. Thesis, University of Luxembourg, 27 Apr., 2015.

[14] A. K. Khandani and P. Kabal, "Shaping Multidimensional Signal Spaces-Part I: Optimum Shaping, Shell Mapping", IEEE Trans. Inf. Theory, vol. 39, n.6, pag. 1799-1808, Nov. 1993.

[15] K. Kobayashi, T. Oka, N. Nakamoto, K. Yano and H. Ban, Polypolarization Multiplexing Scheme for Satellite Communications, 30th AIAA International Communications Satellite Systems Conference (ICSSC) 2012.

[16] DVB-TM-S2 Channel Model Group, DVB-Sx Channel Models, available at: http:www.dvb.org, TM-S2 Working Group, uploaded on 18/01/2013, Document ID: TM-S20125. 